International Journal on Cybernetics \& Informatics ( IJCI) Vol.2, No.1, February 2013

\title{
SLiding Mode Controller Design FOR THE ANTI-SYNCHRONIZATION OF HYPERCHAOTIC LÜ SYSTEMS
}

\author{
Sundarapandian Vaidyanathan ${ }^{1}$ \\ ${ }^{1}$ Research and Development Centre, Vel Tech Dr. RR \& Dr. SR Technical University \\ Avadi, Chennai-600 062, Tamil Nadu, INDIA \\ sundarvtuegmail.com
}

\begin{abstract}
Anti-synchronization of chaotic systems is a research problem that deals with synchronizing the states of master and slave systems at a phase of 180 degrees, i.e. the states are equal in magnitude but opposite in sign. This paper deals with the anti-synchronization research problem of identical hyperchaotic Lü systems (Zhao and Lü, 2008) and new results are derived via sliding mode control. This paper constructs an antisynchronizing sliding controller for hyperchaotic Lü systems based on a general result. MATLAB simulations have been shown to illustrate our sliding controller design for hyperchaotic Lü systems.
\end{abstract}

\section{KEYWORDS}

Sliding Mode Control, Anti-Synchronization, Chaotic Systems, Hyperchaotic Lü System.

\section{INTRODUCTION}

A deterministic chaotic system is a nonlinear dynamical system having properties such as sensitivity to initial conditions and sustained irregularity. Chaos theory and chaos synchronization find many applications in quantum physics [1-2], population biology [3], chemical systems [4], secure communications [5-6], etc.

In the anti-synchronization of chaotic systems, the states of the master and slave systems will have asymptotically the same magnitude, but opposite signs. Anti-synchronization of chaotic systems is an important problem in the chaos literature.

There have been many important methods developed in the literature for chaos synchronization such as active and adaptive control methods [7-10], sampled-data feedback method [11], backstepping method [12], sliding mode control method [13], time-delay method [14], etc.

We have studied the problem of anti-synchronization of chaotic systems in this paper and developed new SMC results for the anti-synchronization of hyperchaotic Lü systems ([15], 2008). MATLAB simulations have been shown to verify and demonstrate the SMC results for the antisynchronization of hyperchaotic Lü systems.

\section{SMC RESULTS FOR ANTI-SYNCHRONIZATION OF CHAOTIC SYSTEMS}

Consider the master system described by the dynamics

$$
\dot{x}=A x+f(x)
$$

where $x \in R^{n}$ is the state of the system and $f: R^{n} \rightarrow R^{n}$ is the nonlinear part of the system.

We take the system (1) as the master system.

DOI : 10.5121/ijci.2013.2105 
Consider the slave system described by the dynamics

$$
\dot{y}=A y+f(y)+u
$$

where $y \in R^{n}$ is the state of the system and $u \in R^{m}$ is the controller to be designed.

We define the anti-synchronization error as

$$
e=y+x \text {, }
$$

Then the error dynamics is obtained as

$$
\dot{e}=A e+\eta(x, y)+u,
$$

where

$$
\eta(x, y)=f(y)+f(x)
$$

We solve the anti-synchronization problem as follows.

First, we take the control $u$ as

$$
u=-\eta(x, y)+B v
$$

where $B$ is chosen so that the pair $(A, B)$ is completely controllable.

Next, we substitute (6) into (4).

Then the error dynamics simplifies to

$$
\dot{e}=A e+B v
$$

which is a single-input, linear time-invariant control system.

In the SMC design, the sliding variable is defined as

$$
s(e)=C e=c_{1} e_{1}+c_{2} e_{2}+\cdots+c_{n} e_{n}
$$

where $C$ is to to be determined.

In SMC design, the motion of the system (7) is restricted to the sliding manifold defined by

$$
S=\left\{e \in R^{n}: s(e)=0\right\}
$$

which must be invariant under the flow of the error dynamics (7).

When in sliding manifold $S$, the error system (7) must satisfy the two conditions:

$$
s(e)=0
$$

and

$$
\dot{s}(e)=0
$$

We use the equations (7) and (8) to rewrite the equation (10) as

$$
\dot{s}(e)=C[A e+B v]=0
$$

We solve (11) for $v$ and obtain the equivalent control law

$$
v_{\text {eq }}(t)=-(C B)^{-1} C A e(t)
$$

where $C$ is chosen such that $C B \neq 0$.

Next, we substitute (12) into equation (7).

Thus, we obtainthe closed-loop dynamics as

$$
\dot{e}=\left[I-B(C B)^{-1} C\right] A e
$$

Next, we choose $C$ such that the matrix $\left[I-B(C B)^{-1} C\right] A$ is Hurwitz.

Hence, by Lyapunov stability theory, the closed-loop error system (13) is globally asymptotically stable.

A sliding mode controller for (7) is obtained as follows.

We apply the constant plus proportional rate reaching law

$$
\dot{s}=-q \operatorname{sgn}(s)-k s
$$

where the gains $q>0, k>0$ are found so that the sliding motion will occur. 
By combining the equations (11) and (14), we can obtain the control $v(t)$ as

$$
v(t)=-(C B)^{-1}[C(k I+A) e+q \operatorname{sgn}(s)]
$$

i.e.

$$
v(t)= \begin{cases}-(C B)^{-1}[C(k I+A) e+q], & \text { if } s(e)>0 \\ -(C B)^{-1}[C(k I+A) e-q], & \text { if } s(e)<0\end{cases}
$$

Theorem 2.1. Consider the master system (1) and the slave system (2). These chaotic systems are globally and asymptotically anti-synchronized by the SMC law

$$
u(t)=-\eta(x, y)+B v(t)
$$

where $v(t)$ is defined by (15) and the sliding mode gains $k, q$ are positive.

Proof. The proof is carried out using Lyapunov stability theory.

First, we substitute (17) and (15) into the error dynamics (4).

Then we get the closed-loop system as

$$
\dot{e}=A e-B(C B)^{-1}[C(k I+A) e+q \operatorname{sgn}(s)]
$$

Consider the candidate Lyapunov function given by

$$
V(e)=\frac{1}{2} s^{2}(e)
$$

which is a positive definite function on $R^{n}$.

We differentiate $V$ along the trajectories of (18) or the equivalent dynamics (14).

Thus, we get

$$
\dot{V}(e)=s(e) \dot{s}(e)=-k s^{2}-q \operatorname{sgn}(s) s
$$

which is a negative definite function on $R^{n}$.

Thus, the error system (18) is globally asymptotically stable by Lyapunov stability theory [16]. This completes the proof.

\section{Anti-Synchronization of Identical Hyperchaotic LÜ Systems VIA SLIDING MOdE CONTROL}

In this section, we derive new results for the anti-synchronization of identical hyperchaotic Lü ([35], 2008) systems using the main result derived in Section 2. Then we shall illustrate numerical simulations of the sliding controller design using MATLAB.

\subsection{Theoretical Results}

The hyperchaotic Lü dynamics is given by

$$
\begin{aligned}
& \dot{x}_{1}=a\left(x_{2}-x_{1}\right)+x_{4} \\
& \dot{x}_{2}=-x_{1} x_{3}+b x_{2} \\
& \dot{x}_{3}=x_{1} x_{2}-c x_{3} \\
& \dot{x}_{4}=x_{1} x_{3}+d x_{4}
\end{aligned}
$$

where $x_{1}, x_{2}, x_{3}, x_{4}$ are states and $a, b, c, d$ are positive, constant parameters of the system.

This is considered as the master system. 
International Journal on Cybernetics \& Informatics ( IJCI) Vol.2, No.1, February 2013

The controlled hyperchaotic Lü dynamics is given by

$$
\begin{aligned}
& \dot{y}_{1}=a\left(y_{2}-y_{1}\right)+y_{4}+u_{1} \\
& \dot{y}_{2}=-y_{1} y_{3}+b y_{2}+u_{2} \\
& \dot{y}_{3}=y_{1} y_{2}-c y_{3}+u_{3} \\
& \dot{y}_{4}=y_{1} y_{3}+d y_{4}+u_{4}
\end{aligned}
$$

where $y_{1}, y_{2}, y_{3}, y_{4}$ are states and $u_{1}, u_{2}, u_{3}, u_{4}$ are the controllers to be designed.

This is considered as the slave system.

The four-dimensional Lü systems (21) and (22) are found hyperchaotic when

$$
a=36, b=20, c=3, d=1
$$

Figure 1 illustrates the strange hyperchaotic attractor of the hyperchaotic Lü system.

The chaos anti-synchronization error is defined by

$$
\begin{aligned}
& e_{1}=y_{1}+x_{1} \\
& e_{2}=y_{2}+x_{2} \\
& e_{3}=y_{3}+x_{3} \\
& e_{4}=y_{4}+x_{4}
\end{aligned}
$$

The error dynamics is easily obtained as

$$
\begin{aligned}
& \dot{e}_{1}=a\left(e_{2}-e_{1}\right)+e_{4}+u_{1} \\
& \dot{e}_{2}=b e_{2}-y_{1} y_{3}-x_{1} x_{3}+u_{2} \\
& \dot{e}_{3}=-c e_{3}+y_{1} y_{2}+x_{1} x_{2}+u_{3} \\
& \dot{e}_{4}=d e_{4}+y_{1} y_{3}+x_{1} x_{3}+u_{4}
\end{aligned}
$$
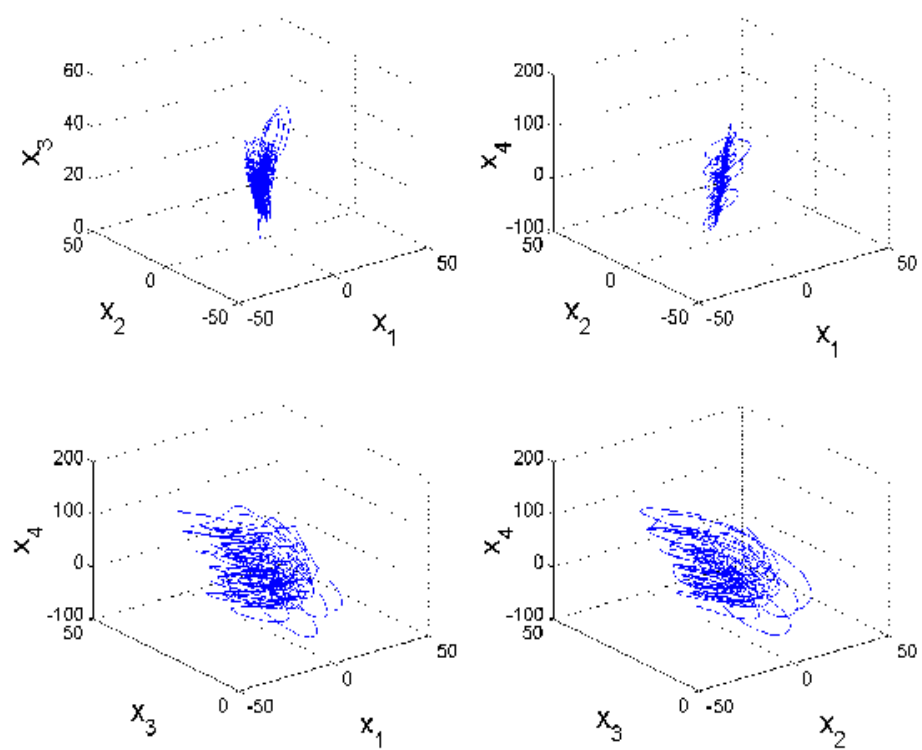

Figure 1. Strange Attractor of the Hyperchaotic Lü System

In matrix form, we write the error system (24) as

$$
\dot{e}=A e+\eta(x, y)+u
$$


where

$$
A=\left[\begin{array}{cccc}
-a & a & 0 & 1 \\
0 & b & 0 & 0 \\
0 & 0 & -c & 0 \\
0 & 0 & 0 & d
\end{array}\right], \quad \eta(x, y)=\left[\begin{array}{c}
0 \\
-y_{1} y_{3}-x_{1} x_{3} \\
y_{1} y_{2}+x_{1} x_{2} \\
y_{1} y_{3}+x_{1} x_{3}
\end{array}\right] \text { and } u=\left[\begin{array}{l}
u_{1} \\
u_{2} \\
u_{3} \\
u_{4}
\end{array}\right] .
$$

In the SMC design, we first set $u$ as

$$
u=-\eta(x, y)+B v
$$

where $B$ is taken so that $(A, B)$ is controllable.

We choose $B$ as

$$
B=\left[\begin{array}{l}
1 \\
1 \\
1 \\
1
\end{array}\right]
$$

The parameter values are chosen as in the hyperchaotic case, viz.

$$
a=36, b=20, c=3, d=1
$$

The sliding mode variable is selected as

$$
s=C e=\left[\begin{array}{llll}
-2 & 10 & 1 & -5
\end{array}\right] e=-2 e_{1}+10 e_{2}+e_{3}-5 e_{4}
$$

which makes the SMC state dynamics globally asymptotically stable.

We take the sliding mode gains as

$$
k=6 \text { and } q=0.1 \text {. }
$$

From Eq. (15), we can obtain $v(t)$ as

$$
v(t)=-15 e_{1}-47 e_{2}-0.75 e_{3}+9.25 e_{4}-0.025 \operatorname{sgn}(s)
$$

Thus, we obtain the required SMC controller as

$$
u=-\eta(x, y)+B v
$$

where $\eta(x, y), B$ and $v(t)$ are given by the equations (26), (28) and (30).

By Theorem 2.1, we arrive at the following result.

Theorem 3.1. The SMC law given by (31) renders the pair of identical hyperchaotic Lü systems (21) and (22) globally and asymptotically anti-synchronized for all initial conditions

\subsection{Numerical Results}

The numerical simulations for the hyperchaotic Lü systems (21) and (22) with the sliding mode controller $u$ given by (31) were plotted using MATLAB.

We take the parameter values as in the hyperchaotic case, viz.

$$
a=36, b=20, c=3, d=1
$$

The sliding mode gains are chosen as

$$
k=6 \text { and } q=0.1 \text {. }
$$

We take initial values of the drive system (21) as

$$
x_{1}(0)=20, x_{2}(0)=-15, x_{3}(0)=-14, x_{4}(0)=12
$$

We take the initial values of the response system (22) as

$$
y_{1}(0)=-5, x_{2}(0)=-25, x_{3}(0)=6, x_{4}(0)=30
$$

Figure 2 illustrates the anti-synchronization of the hyperchaotic Lü systems (21) and (22). 
International Journal on Cybernetics \& Informatics ( IJCI) Vol.2, No.1, February 2013

Figure 3 illustrates the time-history of the anti-synchronization errors $e_{1}, e_{2}, e_{3}, e_{4}$.
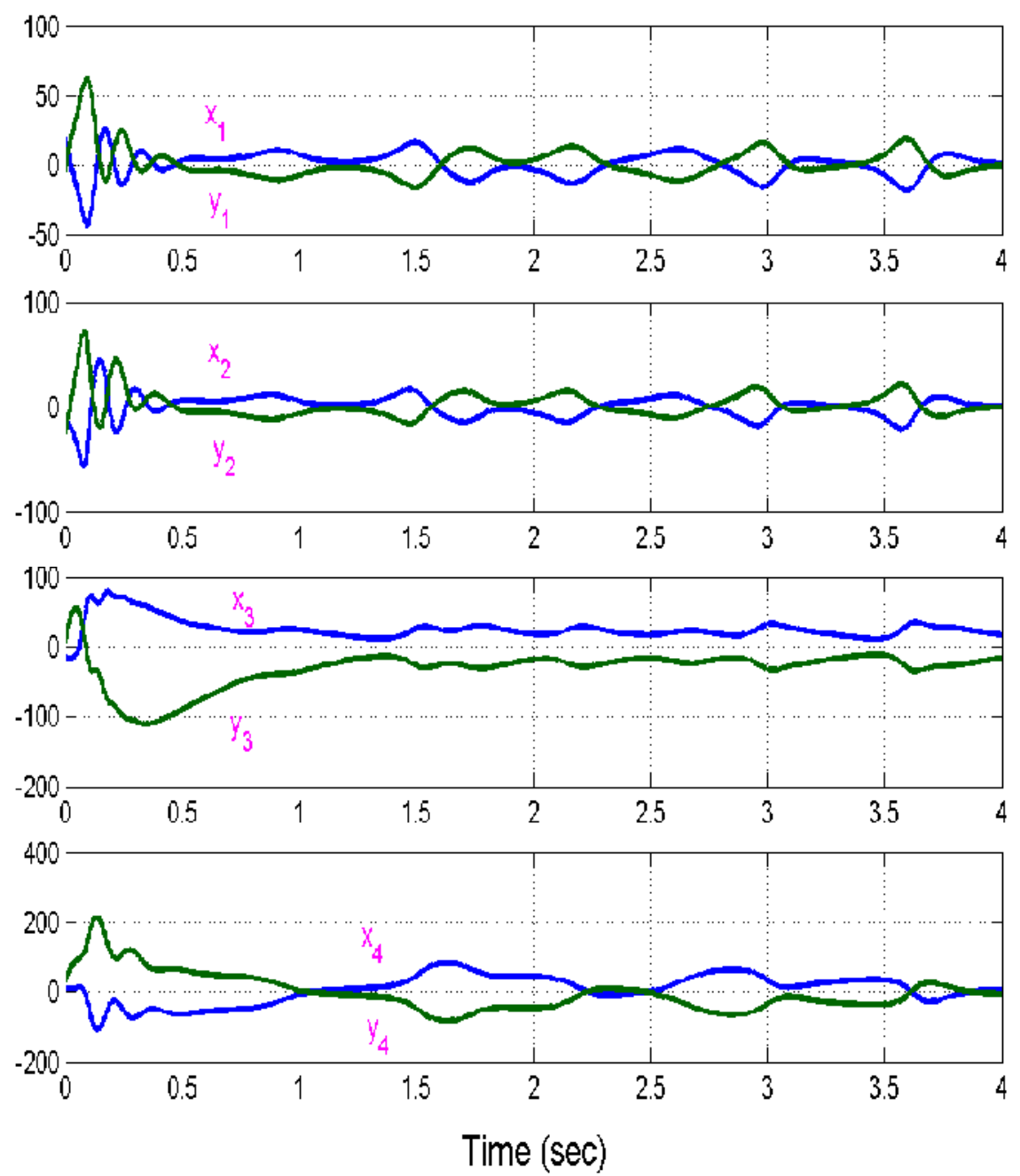

Figure 2. Anti-Synchronization of Identical Hyperchaotic Lü Systems 


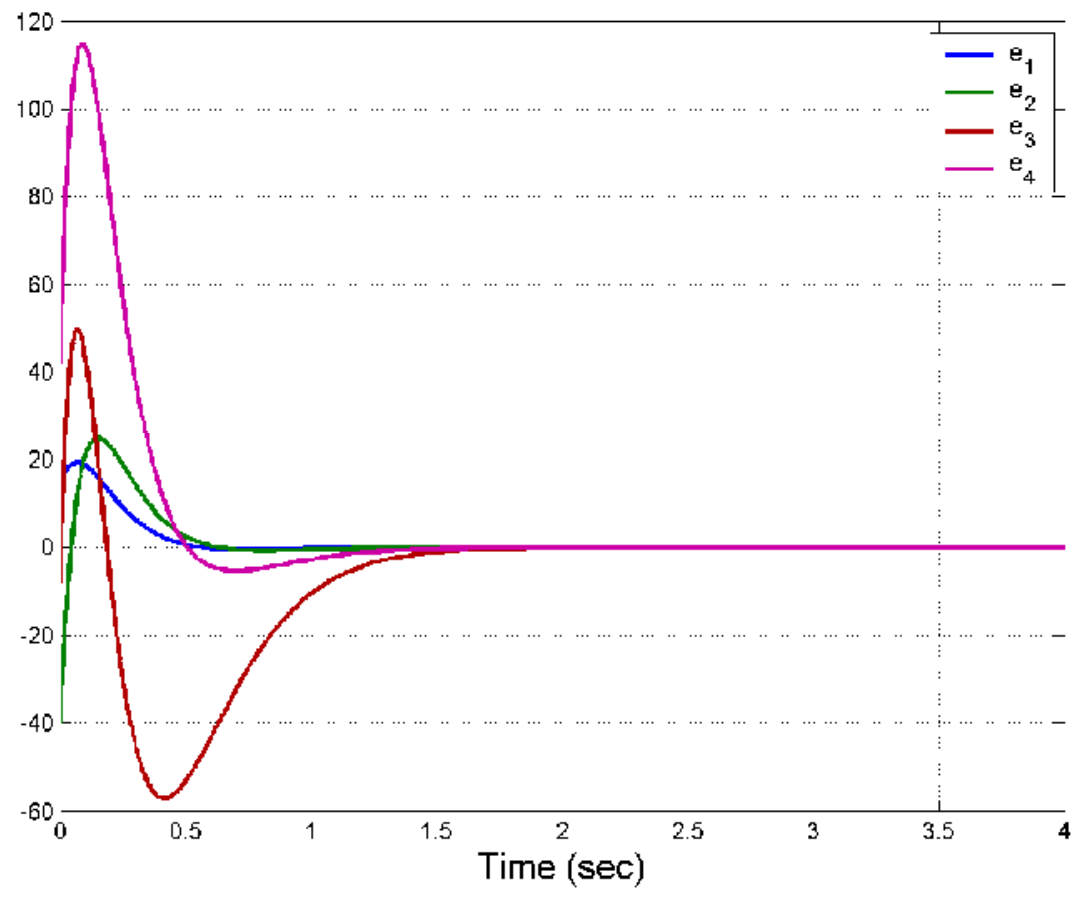

Figure 3. Time-History of the Anti-Synchronization Error

\section{Conclusions}

This paper studied the anti-synchronization problem of chaotic systems using SMC and derived new results for the anti-synchronization for the identical hyperchaotic Lï systems (2008). MATLAB simulations have been shown to validate and demonstrate the new results for the antisynchronization of identical hyperchaotic Lü systems (2008).

\section{REFERENCES}

[1] Efetov, K. (1995) "Supersymmetry in quantum chaos and mesoscopic physics," Physica D: Nonlinear Phenomena, Vol. 83, pp 151-162.

[2] Saif, F. (2005) "Classical and quantum chaos in atom optics," Physics Reports, Vol. 419, No. 6, pp 207-258.

[3] Engbert, R. \& Drepper, F.R. (1994) "Chance and chaos in population biology - models of recurrent epidemics and food chain dynamics," Chaos, Solitons \& Fractals, Vol. 4, No. 7, pp 1147-1169.

[4] Lazzouni, S.A., Siewe, S., Kakmeni, F.M.M. \& Bowong, S. (2006) "Slow flow solutions and chaos control in electromagnetic seismometer system," Chaos, Solitons \& Fractals, Vol. 29, No. 4, pp 988-1001.

[5] Marino, I.P., Lopez, L. \& Sanjuan, M.A.F. (2002) "Channel coding in communications using chaos," Physics Letters A, Vol. 295, No. 4, pp 185-191.

[6] Imai, Y., Murakawa, H. \& Imoto, T. (2003) "Chaos synchronization characteristics in erbiumdoped fiber laser systems," Optics Communications, Vol. 217, pp 415-420. 
International Journal on Cybernetics \& Informatics ( IJCI) Vol.2, No.1, February 2013

[7] Zhang, Q. \& Lu, J. (2008) "Chaos synchronization of a new chaotic system via nonlinear control," Chaos, Solitons \& Fractals, Vol. 37, No. 1, pp 175-179.

[8] Chen, H.H., Sheu, G.J., Lin, Y.L. \& Chen, C.S. (2009) "Chaos synchronization between two different chaotic systems via nonlinear feedback control," Nonlinear Analysis: Theory, Methods \& Applications, Vol. 70, No. 12, pp 4393-4401.

[9] Lazzouni, S.A., Bowong, S., Kakmeni, F.M.M. \& Cherki, B. (2007) "An adaptive feedback control for nonlinear systems with different order," Communications in Nonlinear Science and Numerical Simulation, Vol. 12, No. 4, pp 568-583.

[10] Guo, R. (2008) "A simple adaptive controller for chaos and hyperchaos synchronization," Physics Letters A, Vol. 372, No. 4, pp 5593-5597.

[11] Zhao, J. \& Lu, J. (2008) "Using sampled-data feedback control and linear feedback synchronization in a new hyperchaotic system," Chaos, Solitons \& Fractals, Vol. 35, No. 2, pp 376-382.

[12] Lin, D., Wang, X., Nian, F. \& Zhang, Y. (2010) "Dynamic fuzzy neural networks modeling and adaptive backstepping tracking of uncertain chaotic systems," Neurocomputing, Vol. 73, pp 28732881.

[13] Sundarapandian, V. (2012) "Global chaos control of hyperchaotic Liu system via sliding control method," International Journal of Control Theory and Applications, Vol. 5, No. 2, pp 117-123.

[14] Shahverdiev, E.M. \& Shore, K.A. (2009) "Impact of modulated multiple optical feedback time delays on laser diode chaos synchronization," Optics Communications, Vol. 282, pp 3568-3572.

[15] Zhao, J. \& Lu, J. (2008) "Using sampled-data feedback control and linear feedback synchronization in a new hyperchaotic system," Chaos, Solitons and Fractals $\square$ Vol. 35, No. 2, pp 376-382.

[16] Hahn, W. (1967) The Stability of Motion, Springer, New York.

\section{Author}

Dr. V. Sundarapandian graduated with D.Sc. degree in Electrical and Systems Engineering from Washington University, St. Louis, Missouri, USA in May 1996. $\mathrm{He}$ is working as Professor and Dean of the R \& D Centre at Vel Tech Dr. RR \& Dr. SR Technical University, Chennai, Tamil Nadu, India. He has published over 300 research articles in international journals. He has published over 180 articles in National and International Conferences. He is the Editor-in-Chief of the AIRCC Journals - IJICS, IJCTCM, IJITCA, IJCCMS and IJITMC. He is also the Editor-inChief of the Wireilla Journals - IJCSITCE, IJACEEE, IJCCSCE, IJCBIC and IJSCMC. His research areas of interest are Control Engineering, Scientific

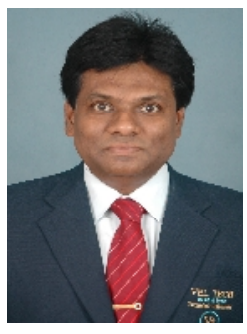
Computing, Chaos Theory, Operations Research and Mathematical Modelling. 